\title{
Special Issue on Soil Microbial Ecology
}

\author{
Haiyan $\mathrm{Chu}^{1,2}$, Yunfeng Yang ${ }^{3}$ \\ 1 State Key Laboratory of Soil and Sustainable Agriculture, Institute of Soil Science, Chinese Academy of Sciences, Nanjing 210008, China \\ 2 University of Chinese Academy of Sciences, Beijing 100049, China \\ 3 State Key Joint Laboratory of Environment Simulation and Pollution Control, School of Environment, Tsinghua University, \\ Beijing 100084, China \\ (C) Higher Education Press 2021
}

Soils would not exist without the activity and diversity of residential microorganisms. Soil microbes are fundamental to maintaining key soil processes associated with organic matter decomposition, nutrient cycling, and plant productivity and are thus integral to human wellbeing. Recent technological advances have exponentially increased our knowledge concerning soil microbial composition, diversity, distribution, and ecological functions. The special issue on soil microbial ecology focuses on various aspects of soil microbiology, including soil microbial spatial distribution, microbial succession, community assembly processes, microbial biogeochemical cycles, the associations between microbial communities and plant health, and the identification and isolation of benefiting microbes for crop growth. The call for papers was widely recognized within the community, reflected by 12 research papers now published in the issue.

We are grateful to all the authors who contributed to this special issue and to many anonymous reviewers who gave critical comments in improving the scientific quality of the manuscripts.
Soil microbial ecology will continuously be a research hotspot in the field of soil biology and microbial ecology. With the development of microbial omics and culture techniques, we will be able to better understand soil microbial distribution pattern and its driving factors, the mechanisms in microbial biogeochemical cycling, and the relationships between soil microbial communities and plant communities, and we will also be able to isolate and culture important microbial species that could be used in agriculture, industry, and medicine.

\section{Guest Editors for the Special Issue:}

Prof. Haiyan Chu Institute of Soil Science, Chinese Academy of Sciences, China hychu@issas.ac.cn

Prof. Yunfeng Yang Tsinghua University, China yangyf@tsinghua.edu.cn 